\title{
Entre psychanalyse et systémique : est-ce que mon cœur balance?
}

\author{
Nicolas Duruz ${ }^{1}$
}

\section{Résumé}

Se référant à son propre parcours psychothérapeutique qui l'a conduit de la psychanalyse à la systémique, sans qu'il n'ait dû renier les apports de sa première formation, l'auteur réfléchit sur les connivences possibles entre ces deux approches, et plus largement sur les possibilités d'une pratique psychothérapeutique non uniréférencée. Selon lui, la diversité des méthodes psychothérapeutiques ne doit pas nécessairement conduire à un éclectisme hypertolérant, souvent confusionnant. Il importe pour le psychothérapeute de se savoir ici plutôt que là, tout en maintenant une ouverture aux autres pratiques grâce à l'intégration assimilative de certaines de leurs techniques.

\begin{abstract}
Refering to his own psychotherapeutical move that took him from psychoanalyze to systemic without having to deny the contributions of his first training, the author discuss the possible connivances between this two approaches and the possibilities of using a non exclusively referenced psychotherapeutic practice. He considers that the diversity of psychotherapeutic methods does not necessarily lead to over tolerant and often confusing eclecticism. It is important that the therapist knows where he positions himself, remaining at the same time open to other practices thanks to the assimilative integration of some of there techniques.
\end{abstract}

\section{Mots-clés}

Psychanalyse - Systémique - Anthropologie clinique - Eclectisme - Intégration assimilative.

\section{Key words}

Psychoanalysis - Systemic - Clinical anthropology - Eclecticism - Assimilative integration

L'autre jour, au terme d'un séminaire que j'animais avec deux collègues sur la psychothérapie individuelle d'orientation systémique (PIOS), un participant, psychiatre bien

\footnotetext{
${ }^{1}$ Professeur honoraire en psychologie clinique de l'Université de Lausanne. Formateur et superviseur à l'Unité d'Enseigmement-CEF de l'Institut universitaire de psychothérapie (Département de Psychiatrie-CHUV), Lausanne. Adresse e-mail : nicolas.duruz@unil.ch
} 
rodé, m'a fait cette remarque : «Au fond, dans votre conception de la PIOS, il n'y a pas de place pour la psychanalyse ! " Le commentaire m'a un peu abasourdi. En effet, tout en me déclarant psychothérapeute systémicien, je considère que ma pensée et ma pratique restent largement nourries par les richesses de la psychanalyse (ma première formation). J'ai même le sentiment d'avoir pu échapper au choix forcé d'un «ou ... ou ... », sans fonctionner pour autant, il est vrai, sur le modèle d'un « et ... et... » intégratif.

Mais qu'en est-il au juste ? Bref retour sur l'itinéraire d'un psychothérapeute systémicien, occasion privilégiée, mais pas entièrement nouvelle, pour réfléchir sur les affinités entre psychanalyse et systémique, et de manière plus générale, sur les possibilités d'une pratique psychothérapeutique non uniréférencée.

\section{Les débuts de la systémique face au bastion psychanalytique}

Aujourd'hui en 2010, nous n'en sommes plus vraiment au temps des clichés, a priori, voire anathèmes que se lançaient facilement psychanalystes et systémiciens dans les années 1970-1980. Je commençais alors ma formation en psychothérapie psychanalytique. Les systémiciens étaient facilement traités de psycho-éducateurs, fonctionnant à la suggestion, raillés pour leurs attitudes de travailleurs sociaux habillés en psychothérapeutes et ignorant tout du fonctionnement psychopathologique des patients. De son côté, la psychanalyse était dénoncée par les systémiciens comme un traitement de luxe, interminable et réservé aux belles névroses, enfermant les analysants dans la solitude du désert ...

Il est vrai qu'à l'époque, la psychanalyse occupait impérieusement le terrain, idéologiquement forte dans les institutions psychiatriques et universitaires en particulier. Les thérapies naissantes - humanistes, TCC et systémiques - d'importation américaine, se frayaient avec peine un chemin dans le paysage des soins en francophonie. Aux systémiciens, il était reproché leur désintérêt pour le sujet et son fonctionnement psychique, son monde imaginaire et fantasmatique, tout accaparés qu'ils se montraient par l'observation des comportements communicationnels dans les interactions familiales. En Suisse romande, je m'en rappelle bien, c'est au contact de la thérapie contextuelle de Boszormenyi-Nagy - la moins comportementaliste parmi tous les courants de la systémique - et en référence à Stierlin - dont la théorie des missions et délégations était très marquée psychanalytiquement - que le bastion psychanalytique se laissa entamer, non insensible à la dimension transgénérationnelle de ces approches, que des avancées psychanalytiques récentes dans le champ de la psychose (Racamier, Guyotat, etc.) exploraient de leur côté par ailleurs. Dans ce contexte, en juin 1978, 
en train d'achever ma thèse sur le narcissisme chez Freud et Szondi à l'aide d'un corpus de 150 tests projectifs de patients border-line, j'ai participé à Florence avec plusieurs collègues psychiatres et psychothérapeutes de Suisse romande au $1^{\text {er }}$ Congrès international de thérapie familiale et systémique en Europe. J'ai eu l'occasion ainsi de voir scintiller les grandes stars du mouvement systémique naissant: Bowen, Minuchin, Boszormeny-Nagy, Whitaker, Watzlawick, Haley, Selvini-Palazzoli, etc.

Rencontres prémonitoires ? J'apprends durant l'été de cette même année que dans le cadre de mon doctorat une bourse pour la relève de l'enseignement m'est allouée, à condition de séjourner aux USA! Je m'y envole fin 1978, dans le but d'approfondir l'approche psychanalytique des états-limites. Par le hasard des chemins et des rencontres, immergé dans un flot coloré de diverses formes de psychothérapie, j'en reviens, deux ans et demi plus tard, avec une formation en systémique acquise à l'Ackerman Institute à New York et un intérêt de recherche pour la psychothérapie comparée. Je suis alors engagé au Centre d'Etude de la Famille à Lausanne, dirigé par le professeur Luc Kaufmann qui a lui-même longtemps travaillé selon une orientation psychanalytique.

\section{L'apport rafraîchissant de la seconde cybernétique}

A ce moment, le courant de la seconde cybernétique, avec ses apports très enrichissants, commence à se déployer. Il faudra toutefois attendre la dernière décade du $20^{\mathrm{ème}}$ siècle pour qu'il ait quelque effet sur les relations entre la psychanalyse et la systémique, mais le climat commence à s'adoucir : qui dit alors systémique, ne dit plus nécessairement anti-sujet, antiémotion, anti-récit. Avec la notion de système auto-poïétique, renvoyant à une organisation interne du système, la «boîte noire » se trouve à nouveau et officiellement ouverte. Très brièvement, car ils sont archi-connus des lecteurs, rappelons trois apports essentiels de la cybernétique de second ordre: ils nous permettront de mieux réaliser comment certaines connivences vont désormais être possibles entre la psychanalyse et la systémique.

Dès les années 70, des biologistes systémiciens (encore eux), tels que Maturana \& Varela (1980), vont insister sur la complexité des systèmes vivants. Relevant l'imprédictibilité de leur évolution, prenant en compte leur inscription dans le temps (« Ce n’est pas nous qui engendrons la flèche du temps. Bien au contraire, nous sommes ses enfants », Prigogine \& Stengers (1979), au point de prêter à chaque cellule vivante une «identité mémoriale », ces scientifiques en viennent à parler de systèmes «auto-poḯtiques », « auto-organisés », ou 
selon l'expression de von Foerster (1981) de «machines non triviales ». Ils visent par ces termes la capacité pour les systèmes vivants, observés dans leur évolution selon un temps diachronique, de générer leurs propres composantes, selon des schémas internes qui leur sont spécifiques. Ils ne sont pas régulés par des «instructions informatives» provenant de l'environnement, avec lequel ils sont toutefois en «couplage structurel». Les notions de «mythe familial», d' «absolu cognitif », de «carte du monde », auxquelles Neuburger (1996), Caillé (1995), Elkaïm (1995) nous ont rendus sensibles dans le champ de la clinique, attestent d'une dimension « invisible » du système.

Cette importance accordée à l' «intérieur » du système va dès lors permettre de mieux comprendre comment dans un système, chaque élément qui le compose a son «autonomie » et l'acquiert selon une dynamique de différenciation. C'est là un apport complémentaire de la cybernétique de second ordre. L'élément n'est plus réduit à ses seuls comportements observables qui alimentent les interactions d'un système. Il est pensé comme disposant d'une vie propre, acquérant une autonomie en lien avec son système d'appartenance, lui-même soumis à un ordre croissant de complexité. En d'autres termes, sa différenciation évolutive va de pair avec celle de son système d'appartenance. Ce qui a pour conséquence que dans un groupe familial, par exemple, chaque membre pourra être reconnu dans sa singularité en fonction de son histoire et de sa trajectoire.

Enfin, à un niveau plus épistémologique, la valeur intrinsèque reconnue à chaque système, comme à chacun de ses éléments constitutifs, génère une compréhension plus élargie de la causalité circulaire. Celle-ci va intégrer désormais l'observateur ou le thérapeute qui, forts de leur propre organisation, sont censés interagir avec le «système observé » et donc l'influencer. Ce n'est plus ce dernier qui est la seule cible d'observation d'interactions circulaires. En prenant au sérieux la part active de l'observateur ou du thérapeute dans ce qu'il observe et ressent - posture exprimée souvent par l'énoncé : «L'observateur entre dans l'univers de son observation »-, on passe d'une épistémologie de la description à une épistémologie de la construction. En termes de processus thérapeutique, on est alors amené tout naturellement à s'interroger sur l'implication personnelle du thérapeute dans le « système observant », implication déterminée par son positionnement aussi bien cognitif que socioémotionnel.

\section{Connivences entre psychanalyse et systémique}


A la lumière de ce bref rappel, on comprend aisément pourquoi la systémique, avec ce nouveau visage, est apparue un peu moins étrangère à la psychanalyse : le symptôme n'est plus considéré dans sa seule valeur comportementale interactionnelle, mais en fonction aussi des processus identitaires du système qui le produisent ; l'individu, même pensé comme système, est restauré dans sa singularité ; son travail d'auto-organisation peut être mis en relation avec sa vie fantasmatique et les avatars psychopathologiques qui l'expriment. En bref, la cybernétique de second ordre réintroduit une dimension subjective et intersubjective dans la dynamique des vivants, ce à quoi la psychanalyse a toujours été très sensible.

De son côté, la systémique, en opérant cet élargissement vers l'«intérieur » n'en reste pas moins fidèle à l'un de ses présupposés: la complexité organisationnelle des ensembles vivants, comme l'a toujours bien relevé Gregory Bateson. En 1970, dans une conférence donnée à la mémoire d'Alfred Korzybski, au cours de laquelle il réfléchit sur ce que signifie la réalité du «soi » et de l' «esprit», il insiste pour que soit pris en compte le double mouvement d'ouverture vers le «dedans » et le «dehors » de l'individu : «La psychologie freudienne, déclare-t-il, a étendu le concept d'esprit vers le dedans, de manière à inclure la totalité du système de communications - les habitudes, l'autodétermination, ainsi que le vaste champ des processus inconscients - à l'intérieur du corps. Ce que je dis, moi, étend l'esprit vers le dehors. Et ces deux mouvements réduisent, l'un et l'autre, le champ du « soi » conscient. Une certaine humilité devient alors de rigueur, tempérée par la dignité ou la joie de faire partie de quelque chose de plus vaste » (Bateson, 1970, pp. 218-219).

Dans ce sens, si l'instrument de la systémique est par excellence, comme l'a relevé justement de Rosnay (1975), le macroscope, cet instrument qui permet de «voir grand », il s'ensuit que la métaphore de la «profondeur», utilisée par la psychanalyse pour signifier la voie royale d'accès à la connaissance de l'homme, avoue ses limites. Elle peut devenir abusive si elle laisse entendre que les psychothérapeutes non psychanalystes sont «superficiels» parce qu'ils ne travailleraient pas en profondeur ... Il s'agirait plutôt de compléter cette métaphore par une vision de l'être humain dans ses dimensions d'extension et de largeur : apprendre à considérer aussi tout ce qu'il y a autour de lui. Pour faire bref, je dirais qu'un homme ne peut se réduire à sa «profondeur»; il est aussi riche de par les nombreuses appartenances qui le font vivre dans la réalité présente. C'est au nom de ces deux regards portés conjointement, que la systémique peut entre autre revendiquer en toute 
légitimité une certaine spécificité dans la pratique d'une psychothérapie individuelle d'orientation systémique (cf. Vaudan et al. 2009) ${ }^{2}$.

Ainsi, pour en terminer avec la cybernétique de second ordre, et comme l'a rappelé à plusieurs reprises Onnis (1991), celle-ci nous invite à substituer à une logique disjonctive en termes de $\mathrm{Ou} \ldots \mathrm{Ou} \ldots$ une logique des articulations dialectiques en termes de $E t \ldots E t \ldots$ Chercher à faire valoir $E t$ le temps synchronique $E t$ le temps diachronique, $E t$ la famille $E t$ l'individu, Et l'externe $E t$ l'interne, $E t$ le système observé $E t$ le système observant, n'est-ce pas là tout l'art de la clinique systémique ?

\section{Place à l'éclectisme?}

Mais ce faisant, au nom de la complexité, la systémique ne cède-t-elle pas à une visée totalitaire, dans son ambition à vouloir tout intégrer? La question mérite d'être posée. En effet, dans le champ de la psychothérapie, une si grande ouverture à des visions et approches différentes pourrait paver le chemin vers un éclectisme confusionnant fort répandu aujourd'hui. N'entend-on pas parler à profusion d'approche multifocale, intégrée, multidimensionnelle, ou de thérapie muldimodale, dialectique, intégrative, etc. ? L'éclectisme est à la mode, et je suis parmi ceux qui s'en méfient: en plus de la fadeur théorique qu'il dégage souvent, je suis indisposé en particulier par la toute-puissance thérapeutique qui l'anime secrètement: rien ne semble résister au psychothérapeute puisqu'il peut toujours trouver dans sa caisse à outils la technique adaptée à la pathologie spécifique de n'importe quel patient ${ }^{3}$. Selon moi, un psychothérapeute ne peut pas répondre à toutes les demandes qui lui sont adressées, s'il est un peu conscient des limites de sa méthode et de sa manière personnelle d'utiliser ses techniques. J'ai développé ailleurs (cf. Duruz, 2009) l'importance pour tout psychothérapeute d'être un peu plus au fait des présupposés ou croyances qui organisent sa pratique et j'y reviendrai brièvement en fin d'article. C'est selon moi le gage

\footnotetext{
${ }^{2}$ On peut relever ici incidemment que l'excellent livre de Neuburger de 1984 : L'autre demande. Psychanalyse et thérapie familiale systémique, est un ouvrage qui porte la marque de l'évolution des relations entre la psychanalyse et la systémique. Montrant qu'il existe des situations où la manière dont le patient vit son problème requiert une thérapie systémique de couple ou de famille et non une psychanalyse - c'est le cas lorsque le patient n'est pas sujet de sa demande, c'est-à-dire qu'il n'est pas à lui seul celui qui porte le symptôme, exprime une souffrance et demande de l'aide -, Neuburger cherche ainsi à légitimer la pratique systémique, parallèlement à la psychanalyse. Mais à cette époque, et dans cet ouvrage, l'apanage de la psychanalyse pour les thérapies individuelles reste encore incontesté.

3 Une toute-puissance semblable se retrouve chez les psychothérapeutes qui se situent à l'opposé de l'éclectisme, à savoir ceux qui se laissent enfermés dans une pensée d'école, les " puristes », qui n'hésitent pas à coucher tout patient sur le lit procustien de leur méthode et de leurs techniques.
} 
d'un renoncement à une toute-puissance narcissique et d'un dialogue possible avec des collègues qui ne travaillent pas exactement comme soi.

Si de par sa généreuse ouverture sur la complexité des processus vivants la systémique peut donc favoriser par certains côtés la voie de l'éclectisme, il me semble qu'elle est en même temps bien outillée épistémologiquement pour la contrer : sa référence entre autres à la théorie des types logiques et son insistance sur la nécessité de toujours dégager les contextes pertinents dans tout travail de communication lui permettent de penser les différences, les limites et les frontières des « choses ». Haro donc sur un éclectisme œcuménique ... baveux ! Pour appui, ce succulent métalogue de Bateson (1953-70), intitulé «Pourquoi les choses ontelles des contours? », dans lequel il dénonce l'hypertolérance. Un père cherche à répondre à sa fille en train de dessiner et qui se questionne sur la nécessité pour les choses d'avoir un contour. Ayant évoqué la position de William Blake, un peintre et poète anglais du 18ème siècle finissant (1757-1827), qui rangeait dans "l'école des baveux" tous les artistes qui peignaient comme si les choses n'avaient pas de contour, il s'entend rétorquer par sa fille : «Il n'était pas très tolérant, n'est-ce pas ? ». La réaction du père ne se fait pas attendre :

LE PERE : "Tolérant ? ... je vois. C'est ce qu'on vous tambourine à l'école! Eh bien, non, Blake n'était pas trop tolérant et il ne pensait même pas que la tolérance soit quelque chose de bien; pour lui, la tolérance rend les choses baveuses. Elle estompe les contours et embrouille tout; autrement dit, elle rend tous les chats gris et, à cause d'elle, plus personne ne peut voir clairement et distinctement.

LA FILLE : Oui Papa ...

LE PERE : Ah non! Ce n'est pas une réponse, « Oui Papa ». Ça prouve tout juste que tu n'as pas d'opinion, que tu te fous éperdument de ce que je dis ou de ce que disait Blake, et que l'école t'a tellement enfumé la tête, avec ses discours sur la tolérance, que tu n'es même pas capable de voir la différence entre deux choses » (p. 47).

\section{L'intégration assimilative}

N'est-ce pas là une bonne mise en garde que tout psychothérapeute qui veut maintenir une ouverture avec d'autres modèles devrait prendre au sérieux : ne pas céder à des mixages opportunistes et peu réfléchis ? En vue de penser plus avant l'objet qui nous concerne - à savoir l'articulation psychanalyse-systémique, et plus largement celle entre plusieurs modèles 
psychothérapeutiques - j'aimerais mentionner un excellent article de Stanley B. Messer (1992), d'orientation psychanalytique, que j'ai découvert, il y a 10 ans environ, intitulé Les croyances des psychothérapeutes intégratifs et éclectiques : un examen critique. Il a été publié dans un ouvrage collectif américain consacré à la psychothérapie intégrative, au sein duquel Messer tient une position très nuancée, plutôt en retrait par rapport à celles des autres auteurs. En clair, il ne croit pas à la psychothérapie intégrative. J'étais bien sûr heureux de repérer, au cœur de la vague déferlante de l'éclectisme américain ${ }^{4}$, une voie présentant des arguments précis et rigoureux, proches de ceux que j'avançais. Messer ne condamne pas du tout le fait qu'un psychothérapeute ou qu'un courant psychothérapeutique s'ouvre à d'autres méthodes pour lui emprunter certaines de ses techniques, mais il dénonce les amalgames faciles qui s'y font alors, au nom d'un soi-disant langage commun neutre. Selon lui, les concepts forgés par chaque modèle, et les techniques qui en découlent, constituent un langage, on pourrait dire un discours, déterminé par des croyances spécifiques. Messer présente dans son article différentes «visions de la réalité ou formes mythiques en psychothérapie », à coloration davantage romantique, ironique, tragique ou comique. Chaque orientation psychothérapeutique tend à privilégier selon lui l'une, voire deux d'entre elles combinées, qui garantissent à cette orientation sa cohérence finale. Fort de ce présupposé, il s'intéresse alors à savoir ce que devient une technique spécifique lorsqu'elle est assimilée par une autre orientation, marquée par une vision philosophique différente. Ce phénomène d'enrichissement d'un modèle par un autre, Messer le qualifie d'intégration assimilative et l'analyse en défendant une position contextualiste. Cela signifie pour lui qu'une technique extraite de son orientation d'origine va nécessairement prendre un nouveau sens en fonction de la structure de l'orientation et de la méthode qui l'adoptent. «Dans l'intégration assimilative, écrit-il, on s'approprie la technique empruntée en l'insérant dans son approche théorique préférée. L'intégration assimilative évite le dogmatisme en reconnaissant la valeur des pratiques des autres, tout en conservant une relative cohérence à sa propre théorie et pratique» (p. 169). Il y donne l'exemple de deux assimilations, celle d'une technique gestaltiste (hot seat) par une thérapie comportementale, et celle de pratiques cognitives par la thérapie psychanalytique. Se référant à la littérature qui en traite, il montre clairement que ces techniques importées sont administrées autrement que dans leur cadre originaire, ce qui

\footnotetext{
${ }^{4}$ Pour les avoir étudiés d'assez près, je suis bien conscient que les courants de psychothérapie éclectique ou intégrative ne sont pas identiques; obéissant à des logiques différentes de combinaison des méthodes psychothérapeutiques, ils ont toutefois en commun une prétention à ne rien vouloir perdre ...
} 
permet précisément au thérapeute qui les adopte de maintenir une relative cohérence de son cadre thérapeutique.

\section{Les échanges entre psychanalyse et systémique : un cas d'intégration assimilative}

Il faudrait développer ici la manière dont des psychothérapeutes systémiciens se sont inspirés de certains concepts-clés de la psychanalyse pour mettre en place des méthodes d'investigation et d'intervention spécifiquement systémiques, et inversement aussi. On peut déjà penser aux notions de soi chez Bowen (1984), de culpabilité existentielle chez Boszormenyi-Nagy (1973), de délégation du moi, du ça et du surmoi chez Stierlin (1978), etc. Plus proche de nous, je pense à la célèbre distinction proposée par Elkaïm (1995) entre programme officiel et carte du monde pour analyser la dynamique du lien conjugal. Le conflit entre ces deux «identités » à l'intérieur d'un même individu n'est pas sans faire penser bien sûr au conflit intrapsychique inconscient tel que l'entend la psychanalyse, utilisé essentiellement par Elkaïm pour rendre compte de la relation conjugale bloquée dans une demande paradoxale à double contrainte réciproque. En reprenant à mon compte cette distinction fort pertinente d'Elkaïm dans un article sur le coup de foudre et ses avatars (cf. Duruz, 2005), j'ai parlé d'une dynamique relationnelle entre deux conjoints « divisés » parce que sujets de désir inconscient, selon l'expression de Lacan. On pourrait en dire autant d'une autre notion d'Elkaïm (1995), celle de résonance, dont on devine facilement l'ancrage originaire dans le champ de la psychanalyse, dans les notions de transfert - contre-transfert plus précisément. Mais en même temps - effet d'une intégration assimilative - cette notion opère un dépassement ou une transformation par rapport à celle de contre-transfert. Ce que le psychothérapeute ressent face au patient ne peut pas se réduire à son histoire personnelle, mais indique quelque chose pour le patient, plus exactement a une fonction pour l'équilibre du système thérapeutique co-construit. A l'inverse, si l'on va de la systémique à la psychanalyse, on pourrait mentionner à titre d'exemple l'emprunt aux théories de la communication fait par Paul-Claude Racamier (1984) autour des années 1970-1980 de la notion de paradoxe, pour mieux dégager l'organisation psychodynamique du patient schizophrène. Plus précisément, en parlant de la paradoxalité du fonctionnement psychique des patients touchés dans leur narcissisme primaire, Racamier vise la disqualification par ces sujets de leurs propres représentations, dans l'acte même de leur refuser un statut de conflictualité et d'ambivalence. Dans la même lignée, Didier Anzieu (1975) écrira un article célèbre sur le transfert paradoxal. 
Ces échanges entre psychanalyse et systémique me ramènent à mon propre parcours psychothérapeutique : clinicien formé d'abord à la psychanalyse, pratiquée pendant quelques années sous forme de psychothérapie psychanalytique, j'ai eu l'occasion par la suite, comme évoqué plus haut, de visiter d'autres terres où se pratiquaient des formes de psychothérapie bien différentes. De ce long «voyage» aux USA, je suis revenu «citoyen» d'un autre royaume, celui de la systémique, tout en ayant eu la chance de pouvoir conserver en quelque sorte mon passeport psychanalytique ... En effet, systémicien dans mes repères explicites, j'ai toujours continué à accorder de l'importance dans ma pratique clinique, sans doute à la faveur d'une certaine intégration assimilative, à la dimension dite inconsciente des pensées et gestes de mes patients, comme des miens d'ailleurs: leur mise en relation a ainsi permis une approche très dynamique et très riche des interactions thérapeutiques. L'apport de la psychopathologie psychanalytique m'est aussi resté très précieux pour la compréhension de certains types de personnalité, de leurs comportements défensifs, comme des fonctionnements familiaux, et cela tout particulièrement dans l'esprit de la pathoanalyse ${ }^{5}$, telle que l'a dégagée mon maître, le professeur Jacques Schotte (1990) à Louvain en Belgique.

Inutile de mentionner que la combinaison systémique-psychanalyse n'est bien sûr pas la seule envisageable. Je connais plusieurs de mes collègues qui allient par exemple pratique systémique et approche cognitivo-comportementaliste, gestaltiste ou autre.

\section{L'apport de l'anthropologie clinique}

Il serait intéressant de décrire minutieusement l'itinéraire de ces psychothérapeutes qui, à partir de leur terre natale, se sont aventurés dans des mondes différents. Quelles raisons et motivations les ont conduits à ces « migrations »? Quelle est leur identité de thérapeute actuellement ? Qu'en est-il de ceux qui se déclarent bilingues ? De ceux qui ont quasi abandonné une langue pour une autre ? Ou qui, fidèles à leur langue maternelle, se disent enrichis par la pratique d'une ou deux autres langues? Du point de vue de l'anthropologie clinique que j'ai cherché à développer depuis quelques années, en vue de penser ce

\footnotetext{
${ }^{5}$ En cherchant à conjoindre le meilleur de la psychanalyse, de la psychiatrie traditionnelle et de l'anthropologie phénoménologique, la pathoanalyse consiste à analyser la condition humaine à partir de la pathologie; elle permet au thérapeute de rencontrer dans le patient un être humain comme lui, participant à une même communauté de destin, en ce sens que les troubles qu'il manifeste sont appréhendés comme des réponses exagérées et caricaturales, mais non moins humaines, à des problématiques d'humanisation que thérapeute comme patient se doivent de confronter pour se réaliser.
} 
phénomène de la diversité des orientations en psychopathologie et en psychothérapie (cf. Duruz, 2009), il s'avère que dès le moment où l'on s'intéresse à des orientations différentes de la sienne, on se trouve transformé dans la compréhension de soi-même. En d'autres termes, la connaissance comparée des autres, c'est simultanément la mise en perspective de soi-même. On est donc ainsi loin de la confusion et des opportunismes qu'on peut observer dans certains assemblages entre pensées et pratiques psychothérapeutiques différentes.

L'anthropologie clinique, à visée inévitablement comparative, défend pour l'essentiel deux points cruciaux : d'une part, la nécessité pour chaque psychothérapeute d'être ancré dans une langue psychothérapeutique tout en ayant une ouverture sur les autres et, d'autre part, l'importance d'être au fait sur les présupposés ou croyances qui l'établissent ici plutôt que là. Dans ce sens, une sorte d'esperanto psychothérapeutique me semble représenter un fort appauvrissement pour la psychothérapie en même temps qu'une grande tromperie, étant donné qu'un modèle ou un courant psychothérapeutique finit toujours par l'emporter sur les autres. Par ailleurs, la clarification de nos présupposés, certes partielle - ce qui est par définition implicite n'est pas si aisément explicitable - nous aide à reconnaître les limites de notre méthode ${ }^{6}$.

Personnellement, le besoin d'être au carrefour de plusieurs chemins m'a toujours habité, et je pense que le monde de la systémique, sensible aux appartenances qui structurent toute existence, m'est en cela très «naturel ». En même temps j'ai toujours été passionné par tout ce que l'homme peut inventer et créer quand il transforme au mieux ses dynamiques pulsionnelles ; j'ai ainsi conservé envers la «bonne » psychanalyse, si naturellement ouverte aux productions littéraires, artistiques, linguistiques ou philosophiques de l'être humain, plus qu'une franche sympathie.

En bref, j'ai le sentiment d'être assez bien à ma place dans le courant systémique, qui ne m'a pas obligé à renier entièrement mes premiers amours envers la psychanalyse : c'est pourquoi, entre eux deux, je pense pouvoir dire que mon cœur ne balance pas! Ce qui ne m'empêche pas, dois-je l'avouer en terminant, qu'au plus profond de moi-même je me sens

\footnotetext{
${ }^{6}$ «En nous attachant à une école théorique, écrit la psychanalyste Mc Dougall (1988), nous faisons partie d'une famille, nous sommes moins seuls face aux incertitudes qui nous assaillent tous les jours. (...) L'idéal serait de tenir dans le même respect que les nôtres les théories des autres; cela nous permettrait de mieux percevoir les limites de nos propres modèles et leur prégnance. » (p. 606)
} 
encore attiré par un autre royaume... Mais qui sait si le psychothérapeute en marche que je suis aura vraiment le temps de le visiter?

\section{Références}

ANZIEU D. (1975) : Le transfert paradoxal. De la communication paradoxale à la réaction thérapeutique négative. Nouvelle Revue de Psychanalyse 12 : 49-72.

BATESON G. (1953) : Pourquoi les choses ont-elles un contour? In Vers une Ecologie de l'Esprit. Tome 1, 46-50. Seuil, Paris, 1977.

BATESON G. (1970) : Forme, substance et différence. In : Vers une Ecologie de l'Esprit. Tome 2, 205-222. Seuil, Paris, 1980.

BOSZORMENYI-NAGY I. \& SPARK G. (1973) : Invisible Loyalties. Harper and Row, New York.

BOWEN M. (1984) : La différenciation de soi. ESF, Paris.

CAILlE P. (1995) : Les situations bloquées du divorce. Thérapie familiale 16 (4) : 349-361.

DE ROSNAY J. (1975) : Le macroscope. Vers une vision globale. Seuil, Paris (Coll. Points).

DURUZ N. (2005) : Du coup de foudre à la crise conjugale. Quelques hypothèses de travail. Thérapie familiale 26 (3) : 299-313.

DURUZ N. (2009) : De l'éclectisme à la pensée d'école en psychothérapie : la voie de l'anthropologie clinique. Persp. Psy. 48 (2) : 194-200.

ELKAÏM M. (1995) : Description d'une évolution. In M. Elkaïm (dir.) : Panorama des thérapies familiales (pp. 587-612), Seuil, Paris.

FOERSTER H. VON (1981): Observing systems. Intersystems Publications, Seaside, California.

MATURANA H. \& VARELA F. (1980): Autopoiesis and Cognition. Reidel Publishing Company, Pays-Bas.

MC DOUGALL J. (1988): Quelles valeurs pour la psychanalyse ? Revue Française de Psychanalyse 52 : 585-612.

MESSER S. B. (1992) : Les croyances des psychothérapeutes intégratifs et éclectiques : un examen critique. Trad. fr. In J. C. NORCROSS \& M. R. GOLDFRIED (Eds) : Psychothérapie intégrative (pp.141-174). Desclée de Brouwer, Paris, 1998.

NEUBURGER R. (1984) : L'autre demande. Psychanalyse et thérapie familiale systémique. ESF, Paris. 
NEUBURGER R. (1996) : Le mythe familial. ESF, Paris.

ONNIS L. (1991) : Le renouvellement épistémologique de la thérapie systémique. Influences actuelles sur la théorie et sur la pratique. Thérapie familiale 12 (2) : 99-109.

PRIGOGINE I. \& STENGERS I. (1979): La Nouvelle Alliance : Métamorphose de la science. Gallimard, Paris.

RACAMIER P.-C. (1984) : Les schizophrènes. Payot, Paris.

SCHOTTE J. (1990): Szondi avec Freud. Sur la voie d'une psychiatrie pulsionnelle. De Boeck, Bruxelles.

STIERLIN H. (1978) : Delegation und Familie. Suhrkampf, Francfort.

VAUDAN C., TRIPET B., CORBOZ-WARNERY A. \& DURUZ N. (2009) : Y a-t-il une place pour la psychothérapie individuelle en systémique ? Thérapie familiale 30 (3) : $379-400$. 